Research Article

\title{
Economic Development Ideas of Regional Convergence Industry Based on Artificial Intelligence Multimedia Background
}

\author{
Zhong Chen ${ }^{1}$ and Yishu Liu (iD) ${ }^{2}$ \\ ${ }^{1}$ School of Economics and Management, Nanchang Normal College of Applied Technology, Nanchang 330108, Jiangxi, China \\ ${ }^{2}$ Business school, Xiamen Institute of Technology, Xiamen 361000, Fujian, China \\ Correspondence should be addressed to Yishu Liu; pim_lau@163.com
}

Received 2 September 2021; Revised 12 October 2021; Accepted 21 October 2021; Published 3 November 2021

Academic Editor: Sang-Bing Tsai

Copyright $\odot 2021$ Zhong Chen and Yishu Liu. This is an open access article distributed under the Creative Commons Attribution License, which permits unrestricted use, distribution, and reproduction in any medium, provided the original work is properly cited.

\begin{abstract}
Today, with the development of the times and the advancement of science and technology, informatization has gradually become an important standard for measuring the state of national development in the course of national development. The artificial intelligence multimedia technology has been developed under the background of informationization and has gradually become an important means to achieve national development and regional economic progress. The impact often depends not only on the economic attributes of the industry but also on the economic attributes of urban planning. Countries and regions with high levels of regional integration and industrial economic development have begun to inject economic knowledge and methods into regional integration and industrial economic processes, which are used to solve or alleviate problems and contradictions in the process of urban and rural construction and development. It can be seen that industrial economy and regional integration are not only a spatial phenomenon but also an economic phenomenon. Based on the needs of the development of urban planning discipline theory and the era of national industrial economic integration, this paper puts forward the research methods and ideas of this paper and combines current multimedia backgrounds and their development trends to analyze key innovations and research frameworks, incorporating the main theoretical research elements into the concept of "combination" of traditional Chinese philosophy, aiming at the main problems and contradictions in the process of China's industrial economic development, respectively, the cultural economy and industrial economy, and the integration of public economy and environmental economy, land economy, and housing economy, a new urbanization development strategy of population economy and regional economic competition.
\end{abstract}

\section{Introduction}

In the current technological environment, the degree of informatization and the resulting multimedia application effects are the basic measures for the development of cities and even the country. Regional economic integration under the influence of multimedia can not only promote the development of the region, but also enhance its competitiveness in other areas, promoting the emergence of emerging industries and strengthening economic development and integration. Multimedia technology has developed in the information age, and it has standards that should be developed at all times. Facing the general trend of regional economic integration, it has made unprecedented contributions and functions for economic development and regional economic integration. This paper studies the regional economic choice methods on the future development path under the background of regional integration, multimedia environment, and information age. The embryonic form of regional economic integration can be traced back to 1921, when Belgium and Luxembourg formed an economic alliance, and later the Netherlands joined to form the Benelux economic alliance. In 1932, the United Kingdom and the Commonwealth member states formed a preferential tariff zone in the British Empire. The member states reduced tariffs to each other, but they still maintained the original higher tariffs for countries that were not members of the Commonwealth, forming a 
preferential tariff zone. The most representative one is the European Union.

Based on the importance of regional integration of industrial economy, many research teams have carried out research, analyzed its importance, necessity, and development prospects, and achieved good results. Fang [1] expounded the concept of smart city and regional industrial economy, then analyzed the status quo of smart city and industrial economy with the concept of sustainable economic development, and deeply analyzed the shortcomings of industrial economic development. Finally, with "innovation and development" as the main line, the path choice of promoting regional industrial economic integration in the construction of smart cities is explored. Cao [2] proposed that the smart city industrial economy is mainly built on the basis of the Internet of things, cloud computing, and the Internet. When the city develops, the data information of each link is integrated, and the city can be intelligently processed, strengthening the management and control of the city. Niu [3] mainly analyzes the development and construction of smart cities and industrial economic prospects and also discusses aspects such as integrated development in economic development. Xia and Chen [4] mentioned that urban planners should promote the construction and improvement of the information platform and use the existing infrastructure to achieve efficient integration of resources at the two levels of smart city construction and regional industrial economic development, eliminate the gaps and differences between various departments and enterprises, use modern digital technology to promote the city's comprehensive coverage of digital resources, establish a scientific information management system, and ultimately constitute a scientific and effective information platform to build smart cities and provide strong platform support for the integration and development of regional industrial economy. Lin [5] found from the current economic development in China that the interregional economic structure is unreasonable and the adjustment is slow. This phenomenon is another reason that hinders China's economic development. Therefore, it is necessary to formulate a development policy that meets the requirements according to the specific conditions of different regions, clarify the economic development status of the region, promote regional and regional integration, and finally achieve the regional coordinated development. Liu and Cai [6], based on the definition of industrial location advantages and its measurement methods, used the location quotient measure model to analyze the three industrial advantages of Fujian Province, then compare the differences in industrial location advantages of the three provinces along the southeast coast of Fujian, Zhejiang, and Guangdong, and propose enhancing the industrial location advantage of Fujian Province from the aspects of promoting the deep integration of industries between Fujian and Taiwan, docking the Yangtze River Delta Economic Zone, and accelerating the construction of the Fujian Free Trade Zone to promote industrial transformation. Jiang [7] started with the basic relationship between industrial economy and new media and found the best way to integrate the two and the point of convergence to form a new model of industrial economic development. By formulating an institutional model that is consistent with the development of relevant enterprises, the scope of influence of new media will be gradually increased and the overall development level of the industrial economy will be improved. Zhang [8] found that if the ceramic industry can be integrated with other industrial areas, then artistic innovation and economic development can be achieved simultaneously. This requires the integration of at least two related areas to achieve, but the current technological development tells us that this is not impossible. It is hoped that through the research on the development path of Tangshan ceramic art innovation and industrial economy, the revitalization of Tangshan ceramic industry will begin. Dong [9] said that cultural industry, as an important pillar industry of the national economy, can form a fusion with the Internet or the Internet economy in the current social ecology, which is the key to the future direction of the cultural industry. From the integration point between the two, the paper explores the interinfiltration of the Internet industry economy and cultural industry into the content innovation and the future development direction, in order to provide a valuable reference for the industrial integration development. Wang [10] found that local media has played a greater role in promoting regional economic development by integrating resource advantages, making suggestions for regional economic development, and promoting the development and transformation of the media itself, which has become a local media business development. There is urgent need to consider the issue. Wang [11] said that, from the perspective of the current stage of industrial economy, it has become inevitable to use new media to adjust the industrial economic structure and realize the functionalization of scientific and technological information. When new media becomes an important part of the external promotion and branding of the industrial economy, the integration of industrial economy and new media naturally occurs. Wu [12] proposed that informationization brought new vitality to the industrial economy, because informationization promoted the optimization of industrial structure, increased labor productivity, and promoted the growth of industrial economy.

However, some people have analyzed regional integration from different aspects and proposed new explanations and innovative research on their development ideas. Zhao [13] further deepened the construction of the Yangtze River cloud platform, improved the platform's security, scalability, and intelligence level, improved the government service and comprehensive service functions of the Yangtze River cloud platform, improved the user experience, and promoted "News + Government Affairs," "News + Service," "News+ Politics," and "News + E-commerce," and other concepts are implemented. We will hold a special training course for the construction of county-level media center in a timely manner and provide comprehensive training for the heads of the media and media centers, media editors, and technical operators. Others describe integration as the formation of a multicountry economic region in which trade barriers are weakened or eliminated, and production factors tend to flow 
freely. The so-called "region" refers to a geographic scope that can carry out multilateral economic cooperation, which is often larger than the geographic scope of a sovereign country. Zhang et al. [14] found that informatization has formed a certain integration trend with rural regional economic development. For contemporary college students, informationization should be used to participate in rural regional economic development, and efforts should be made to promote rural regional economic development. Cai [15] proposed to promote the integration and development of national sports cultural resources and ethnic regional tourism industry and then increase the cultural connotation of tourism products, which has practical significance. It is also the transformation and upgrading of tourism industry and the realization of tourism-oriented primary stage to cultural tourism, an important way to transform the advanced stage of the main. Qimengya [16] proposed that the new era is a new measure to promote the formation of a new comprehensive opening pattern and is also a new practice to promote the development of "one country, two systems." Guangdong, Hong Kong, and Macao Dawan District actively promote the integration of infrastructure and accelerate the construction of world-class urban agglomerations, create an international science and technology innovation center, and continue to do a good job in reform and opening up. Zhang [17] proposed that the main purpose of the development of higher vocational schools is to promote the development of regional industrial economy $[18,19]$. The educational model of higher vocational schools has a close relationship with the economic industrial structure, facing the continuous development of the economy and effectively integrating higher vocational education and economic industry. Zhu [20] found that a country or region that wants to achieve leading development in the field of science and technology, occupying a favorable position, needs to continuously improve its informationization level from informationization and through informationization and regional economy. Integration will promote the comprehensive development of local comprehensive strength, and informationization will not only promote the development of traditional economy, but also further strengthen regional competitiveness and industrial system construction. Shao et al. [21] and others briefly introduced the specific content of the "One Belt, One Road," analyzed the opportunities brought by the "One Belt, One Road" initiative for regional economic development, and explored the specific path of the "One Belt, One Road" initiative and regional economic integration development. $\mathrm{Yu}$ and Cao [22] promoted the integration of the first, second, and third industries of agricultural parks with the rural complex as a carrier [23], promoted the integration of agriculture, housing, and rural tourism, and enabled the tourism industry to drive the development of modern agriculture and increase farmers' income. Alpine Ice [24] found that regional media integration is at the stage of addition, and there are still problems such as heavy form and light impact, regional development imbalance, lack of organization and system guarantee, and lack of new media quality of practitioners. In this regard, it is suggested that top-level design and institutional mechanism innovation should be strengthened; cross-regional collaborative resonance should be promoted to create a new engine for county-level media integration, develop a sustainable talent strategy, tap the intrinsic value of media data, actively innovate technology applications, and form new ones. Production concepts and production models have promoted breakthroughs in the deep integration of regional media [25].

Multimedia technology has many advantages in the economic development of regional integration industry. Some research teams have carried out a series of researches on the application of multimedia technology and achieved remarkable results. Wang [26] studied the relevant content of regional economic integration under the multimedia development model, proposed a possible future development direction and ideas, and promoted the development of related industries and economy. Regional economic development in the multimedia environment is an important way for modern society to achieve regional economic integration and promote social modernization. Li and Tian [27] said the multimedia network has shown strong vitality, which has promoted the transformation and upgrading of China's economic structure. At the same time, it has changed the country's economic development mode, the production and management methods of enterprises, and the way people live and play [28]. In the new era, in order to better meet the needs of the development of China's economy, multimedia networks must keep pace with the times, so it is necessary to analyze the impact of multimedia networks on the economy. Li [29] said that, for the real economy, it is necessary to strengthen the competitiveness of the real economy and promote the transformation and upgrading of the real economy by strengthening measures such as institutional construction and supervision in the virtual economy, deepening supply-side reforms, and reducing costs. Wu and $\mathrm{Ma}$ [30] found that the scale of postgraduate education has a positive effect on the economic development of the tertiary industry. The scale of master student education has a more obvious effect on the economic development of the tertiary industry and has a faster effect; the scale of graduate education on the economy of the tertiary industry has regional differences in the promotion of development, which have positive effects on the eastern and central regions, but no significant impact on the western regions.

This paper firstly reviews the history and characteristics of western developed countries and developing countries in the process of regional integration and refines and summarizes their experiences and enlightenments through multimedia technology. Secondly, through summarizing the economic characteristics of industrial economy, this paper puts forward the research methods and diversity characteristics of industrial economics theory and attempts to explore the multimedia research framework of urban planning economics. Finally, the main economic elements that promote the economic development of regional integration industries are injected into the "four-in-one" concept of integration, integration, integration, and competition in the development theory system and the combination of cultural economy, industrial economy, and public economy, the integration of environmental economy, 
the integration of land economy and housing economy, and the integration of population economy and regional economy.

\section{Method}

2.1. Regional Fusion Industry Economic Thinking Research Methods. This paper uses a combination of urban planning, geography, sociology, economics, psychology, philosophy, and other theories and methods. A large number of historical documents and statistical data on the economic development of regional integrated industries were collected, analyzed, researched, and combed, and the results were compared. This paper provides effective data and theoretical support for development strategies from the perspective of regional planning industry economics.

2.1.1. Literature Review Method. This paper uses a large number of literature indexes to collect relevant literature and research results of urbanization process in several cases and detailed theoretical and data support for the comparative analysis and summary evaluation of the article and how to develop new urbanization in China and identify the challenges you will face and the risks you should circumvent.

2.1.2. Comparative Research Method. After sorting out the characteristics of regional integration development at home and abroad, it compares the dilemmas encountered by different countries in the process of regional integration and their solutions. Secondly, it compares the characteristics of China's regional integration development and summarizes the experiences and problems of each stage. Finally, through comparative research and systematic analysis, combined with the industrial economic theory research framework, it puts forward the strategic recommendations for regional integration development.

2.1.3. Social Investigation Law. This paper summarizes the outstanding problems and contradictions in the traditional industrial economic process. When answering the summary of key social phenomena, it uses field research, observations, interviews, and other social survey methods to verify and validate. Studying the causes of these prominent contradictions and problems, as well as the actual impact on urban residents and immigrants, laid the foundation for the regional integration of industrial economic development strategies and the recommendations of the relevant theories of industrial economics.

In general, regional economic integration is also called "regional economic grouping." Two or more countries in the same region gradually transfer part or even all of their economic sovereignty, adopt a common economic policy, and form an exclusive economic group. The rapid development of regional economic integration on a global scale mainly depends on three ways: one is to continuously deepen and upgrade the existing form; the second is to expand the existing group members; the third is to conclude a new regional trade agreement or restart the long-lost regional economic cooperation negotiation.

\subsection{Related Theoretical Research}

2.2.1. Mainstream Urban Economics Theory. The main goal of mainstream urban economics research is to maximize the utility of social subjects. Due to the diversification and limited use of urban land, capital, and other resources, the mainstream urban economics community generally believes that, in the process of urbanization development, in most cases, there should be a market to complete resource allocation, but it also needs to be necessary. Urban economics is an economic discipline that studies the economic relations and laws of the entire development process of the city's emergence, growth, and urban-rural integration. Urban economics focuses on the entire historical process and laws of the emergence and growth of cities and finally the integration of urban and rural areas, as well as the various production relations embodied in economic activities inside and outside the city. It is an important guarantee for the realization of regional integration.

2.2.2. Conservative Urban Economics Theory. The founder of Conservation Urban Economics is Milton Friedman, whose representatives include Ronald $\mathrm{H}$. Coase and Friedrich A. Friedman A. Hayek and George Stigler. Conservative urban economics emphasizes that urban economics should pay more attention to social and economic problems such as urban land use and urban transformation in the process of urbanization. It believes that mature economic systems must ensure that members of society can freely engage in economic activities. It should not be too concentrated, and intervention in the market should be limited.

2.2.3. Marxist Urban Economics Theory. In the past half century, Marxist economics has achieved fruitful results. Both economic theory and social thought have had a tremendous impact in the west. However, for a long time after the Second World War, the Marxist economics school was also strongly suppressed in the United States, and the United States was proud of its freedom of speech and academic freedom. It can only adapt to the national conditions according to the background of the times and the actual situation, but the most important theme of class struggle is preserved.

2.2.4. Urban New Economic Growth Theory. The main idea of the theory of urban new economic growth is to internalize scientific and technological progress and believe that endogenous technological progress and innovation are the decisive factors for the sustained growth of urban economy. In addition, the government's policies on industry, trade, and finance play an important role in achieving stable economic growth. 
2.2.5. Pareto Efficiency. In economics, Pareto efficiency (also known as the "Pareto optimality") basic idea is to link the efficiency assessment of a social organization with the welfare of individuals in the organization, and the personal welfare status improvement is seen as a sign of the efficiency of social organizations. Under the premise of production technology, resource elements, and personal preferences established by a certain social organization, when the redistribution of social resources does not make some people's situation worse and when the situation of people is not improved, the resource allocation of social organizations is at its best. If a social organization reconfigures social resources, it can make some people's situation better. It will not affect the situation of any other person but prove that the efficiency of social resource allocation has been improved.

\subsection{Development and Advantages of Business Activities in the Multimedia Environment}

(1) Promoting the generation of virtual goods on the network. With the widespread use of multimedia network technology and computer tools, relatively new forms of goods have emerged, freeing people from the perception of traditional commodity concepts.

(2) Changes in the equilibrium state of supply and demand of goods. Due to the asymmetry of information and the imbalance of current people's needs, the supply and demand of commodities are often in an unbalanced state.

(3) Stimulating enterprises to carry out technological innovation. The development of multimedia networks not only promotes the development of the overall economy, but also promotes the continuous improvement of their own technical conditions.

(4) Reducing costs and increasing profitability. Through multimedia network technology, enterprises can complete the procurement design, production, sales, and other links through the establishment of a good management information system.

(5) Getting more and faster information. From 2015 to 2018, China's rural per capita disposable income rose from 1.8 yuan to 34,000 yuan, and the per capita disposable income of urban population rose from 39,000 yuan to 52,000 yuan, while the average price of mobile phones dropped from about 4,400 yuan in 2015. It will be about 8,900 yuan by 2018 .

(6) Artificial intelligence. Artificial intelligence is a branch of computer science. It attempts to understand the essence of intelligence and produce a new intelligent machine that can react in a similar way to human intelligence. Research in this field includes robotics, language recognition, image recognition, natural language processing and expert systems, etc. Since the birth of artificial intelligence, the theory and technology have become increasingly mature, and the field of application has continued to expand.
It is conceivable that the technological products brought by artificial intelligence in the future will be the "containers" of human wisdom. Artificial intelligence can simulate the information process of human consciousness and thinking. Artificial intelligence is not human intelligence, but it can think like humans and may exceed human intelligence.

\section{Experiment}

\subsection{Analysis of the Characteristics of Regional Integration Industry Economics}

3.1.1. Morphological Characteristics. The geometric characteristics of regional fusion industry economics are mainly reflected in the analysis of social and economic benefits, focusing on the explicit economic expression of regionalbody spatial form. From the macro to the micro, from the whole to the local, from the outside to the inside, from the single-dimensional to multidimensional abstract geometric spatial form and scale, choose the geometry of the most "economic" regional spatial combination. The main content involved includes the size of regional land use, compact and loose, concentrated and dispersed, open and closed. Examples of the multidimensional geometric space form of the road network layout are density, slope, direction, and form.

3.1.2. Technical Characteristics. The technical characteristics of regional integration economics are mainly reflected in the application of economics, such as external analysis, econometric model analysis, statistical analysis, marginal analysis, cost-benefit analysis, and other economic research techniques. Provide theoretical basis and technical support for decision-making in the process of regional integration planning and management. When drawing on the research and demonstration of relevant technologies in regional planning economics, the first thing to pay special attention to is that it cannot be simply copied. It is necessary to fully understand the social, political, and historical background and other factors that form the formation and maturity of technology and make a comprehensive comparative analysis based on their own stage background. Secondly, the technical characteristics of regional integration planning economics are incorporated into the regional integrated planning technology system, supporting the construction of a complete regional planning technical system. The integration method is not particularly complicated. Compared with the literal meaning, it can actually be simplified to integrate the technical characteristics of any technology into the entire technical system. This is a substantial and rich process.

3.1.3. Social Characteristics. With the rapid development of urbanization, the theoretical system of regional planning disciplines has been continuously improved. Judging from the development trend of the subject theory, the regional integration industry economy is increasingly represented as a social work that integrates political, economic, 
geographic, architectural, engineering, technological, environmental, and other elements. It not only affects the improvement of regional core competitiveness, but also directly relates to the coordinated supply of urban residents' welfare. It can be seen that the social characteristics of regional planning economics are assisting the government in the strategic arrangement of regional integration of future development and analyzing the construction of land, municipal facilities, public service facilities, park green space, open space, and other important public resources. The process of coordination and layout has emerged, so that the urban social economy can develop healthily, benignly, and orderly.

\subsection{Analysis of Problems in the Process of Regional Integration Industry Economic Development}

3.2.1. Resource and Energy Shortages Restrict Regional Integration and Development. In the past, China's annual reduction in the amount of cultivated land due to urbanization was close to 20 million $\mathrm{mu}$, which further aggravated the shortage of water and soil resources that were already prominent. From 2015 to 2018, China's urbanization rate will grow at an average annual rate of 3 percentage points, while per capita energy consumption will grow at an annual rate of $7.99 \%$. Based on this, when China's urbanization level reaches $65 \%$, the country's total annual energy consumption will reach 6.4 billion tons of standard coal, breaking the energy consumption limit of China's 4 billion tons of standard coal. According to the research of French economist Sandra Poncet, the average tariff of China's domestic interprovincial merchandise trade reached $46 \%$ in 1997 , a full $11 \%$ increase over 10 years ago. This tariff level exceeds the tariff level among EU member states. It is equivalent to the trade tariff between the United States and Canada. In other words, in 1987, the number of Chinese consumers buying homemade products from their respective provinces was 10 times that of products from other provinces, and by 1997 , this proportion reached 21 times. Therefore, how to break local protectionism and build the overall advantage of the regional economy has become one of the focuses of the current Chinese government and academic circles.

\subsubsection{Ecological Environment Constraints Forced the} Transformation of Industrial Economic Development Model. In 2015, China's total sulfur dioxide emissions were 21.176 million tons, and total nitrogen oxide emissions were 23.378 million tons. In the first half of 2016, the air quality ratio of the Beijing-Tianjin-Hebei region, the Yangtze River Delta, and the Pearl River Delta in 74 regions exceeded the standard, at $35.6 \%$. The regional environment has deteriorated drastically, and the threat of environmental pollution to human health has increased. Therefore, the traditional economic development model is unsustainable, and the industrial economy and regional integration development model needs to be transformed.
3.2.3. The Public Economic Development Is Seriously Lagging Behind. In 2015, China's total energy use was six times that of 1978, and the growth rate of energy use was seriously more than expected. In 2016, China's energy consumption level has reached the level of 2020 predicted six years ago. In addition, the comprehensive carrying capacity of cities and towns is low, so that one-third of the resident population has no access to basic public services such as urban medical care, education, housing security, and social security, which has aggravated social inequality and intensified society. According to statistics, the total public safety expenditure in China reached 514 billion yuan in 2018, which indicates that the economic benefits of regional integration development are offset by higher and higher social costs.

3.2.4. Regional Differences in Public Facilities Are Large. In 2015, the national water supply penetration rate of county towns and townships was less than $80 \%$, and the gas penetration rate of county towns was less than $70 \%$; the standard of urban and rural drainage facilities was low, the density of drainage network was less than $1 / 2$ of that of developed countries, and the flood warning facilities had a low penetration rate. As a result, $62 \%$ of the 351 towns surveyed in China between 2015 and 2018 have experienced internal disasters. Compared with the waste-free treatment technology that has been widely used in western developed countries, the pollution-free treatment rate in China's cities and towns is currently less than $80 \%$, of which only $40 \%$ is in the county. The land occupation problem is serious and it pollutes the ecological environment. In terms of power supply facilities, some urban power grid equipment is aging and there are problems such as high energy consumption and low utilization rate, while power supply reliability in rural areas is poor. The shortage of resources and energy restricts the development of regional integration; the constraints of the ecological environment force the transformation of industrial economic development; the severe lag of public economic development and the large regional differences in public facilities are all remaining problems of the development of traditional regional economic integration. Figure 1 shows an analysis of the problems that occurred in the development from 2015 to 2018.

As can be seen from the figure, from 2015 to 2018, in terms of resources, 2016 was down 2 percentage points from 2015, but it increased by 20 percentage points in 2017 and 12 percentage points in 2015-2018; public economy, in 20152016 , surged by $18 \%$ in the year, but it dropped by $16 \%$ in 2017, but it has been steadily fluctuating throughout the four-year period; in terms of public facilities, it has increased by $25 \%$ in four years, indicating that people's demand for public facilities has greatly increased, and people began to enjoy life and pursue quality of life; in terms of environmental economy, the overall increase of $8 \%$ reflects the improvement of people's environmental needs, but the extent of change is within a reasonable range. After we analyze the problems in the process of regional integration industrial economic development, they can be used as a 


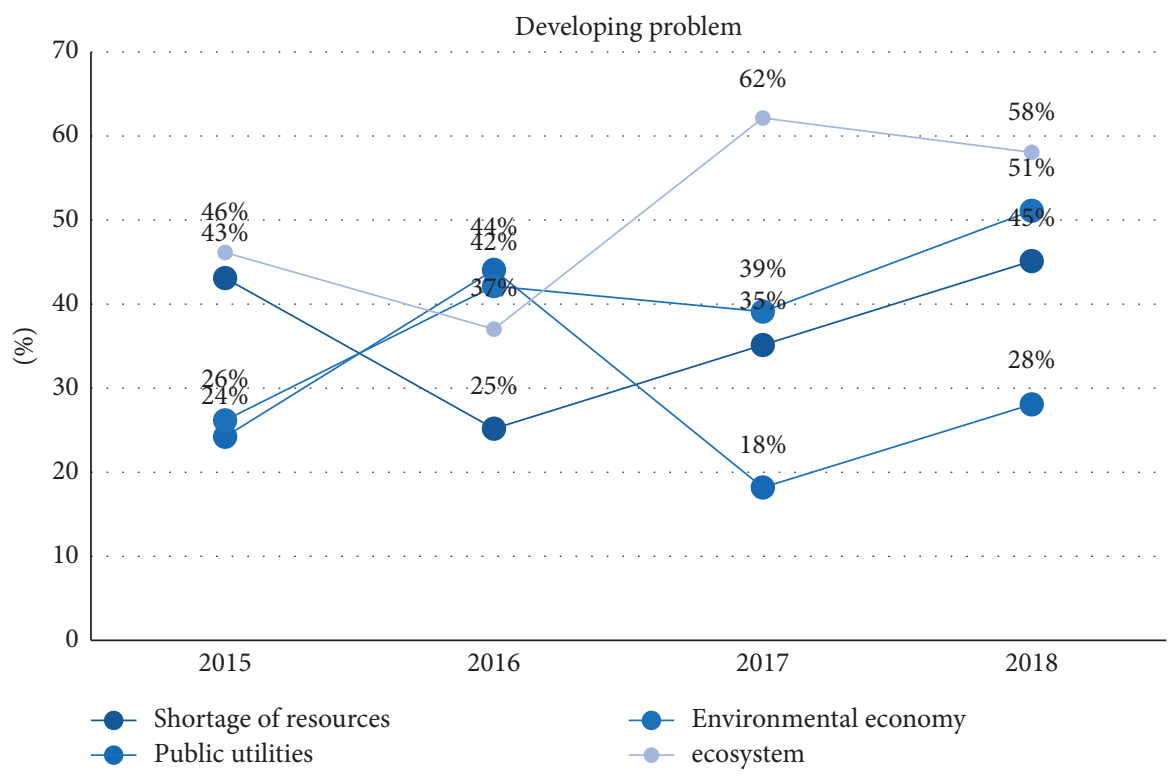

Figure 1: Analysis of the status quo 2015-2018.

reference for this article. We deliberately solve these problems in the experimental research and the solutions to these problems are also relatively diverse in the research results.

\subsection{Implementation Path of Regional Convergence Industrial Economic Coordination Development}

3.3.1. Maintain Regional Cultural Background. With the emergence and reproduction of different nationalities, it has begun to give culture a national character. The local consciousness of regional culture in China is deeply rooted and directly or indirectly affects the operation and concentration of regional local economic capital. At this stage, due to the obvious regional differences in China's economic development, there is a large gap between consumption and income levels among regions. However, as the disparity in disposable income of residents continues to shrink, cultural factors are bound to become the main factors guiding population and economic agglomeration.

3.3.2. Coordinating Regional Cultural Resources. After more than 30 years of rapid development, China's rapid urbanization has not only achieved a historic breakthrough in regional industrial economic construction, but also achieved remarkable results in regional cultural economy. The overall planning of regional cultural resources is the core strategy of using regional cultural resources to promote the overall development of regional industrial economy and is also an important way and method to improve the quality of life of regional residents and enhance the core competitiveness of the region.

3.3.3. Creating a Cultural Industry Space. The planning research on the development of cultural and creative industries has also begun to pay attention to the comprehensive protection and utilization of regional development history and cultural environment, the rational construction of new and old cultural and creative industry space, the production of cultural industry and the rational layout of consumption space, and the economic development of regional cultural industry.

3.3.4. Focus on Regional Cultural Marketing. In addition to reviving the cultural economy, some towns began to conduct regional brand marketing from cultural products, through the hosting of festivals, cultural and sports events, summit forums, and other major events to expand regional integration influence, enhance urban visibility, and shape the image of the town. Figure 2 is an analysis of the economic development path of regional fusion industry.

\section{Results and Discussions}

4.1. Demand Analysis of Regional Integration Industry Economic Development. With the advancement of human science and technology and the needs of its own development, the mode of transportation is also changing. In the entire development process of the world region, changes in the public transportation system and changes in transportation modes have had an important impact on the formation, expansion, form, scale, and structure of regional integration. As the pace of China's industrial economy continues to accelerate, urban and rural transportation will become the main area for the development of urban and rural integration. The following can be said: "economic coordination, traffic first." Only by increasing the support of the public economy for the construction of transportation facilities can we lead the sustainable development of the urban and rural economy and meet the basic needs of regional integrated industrial economic development.

From a macroscopic perspective, regional space is a patch of urban and rural spatial patterns, and towns and villages are 


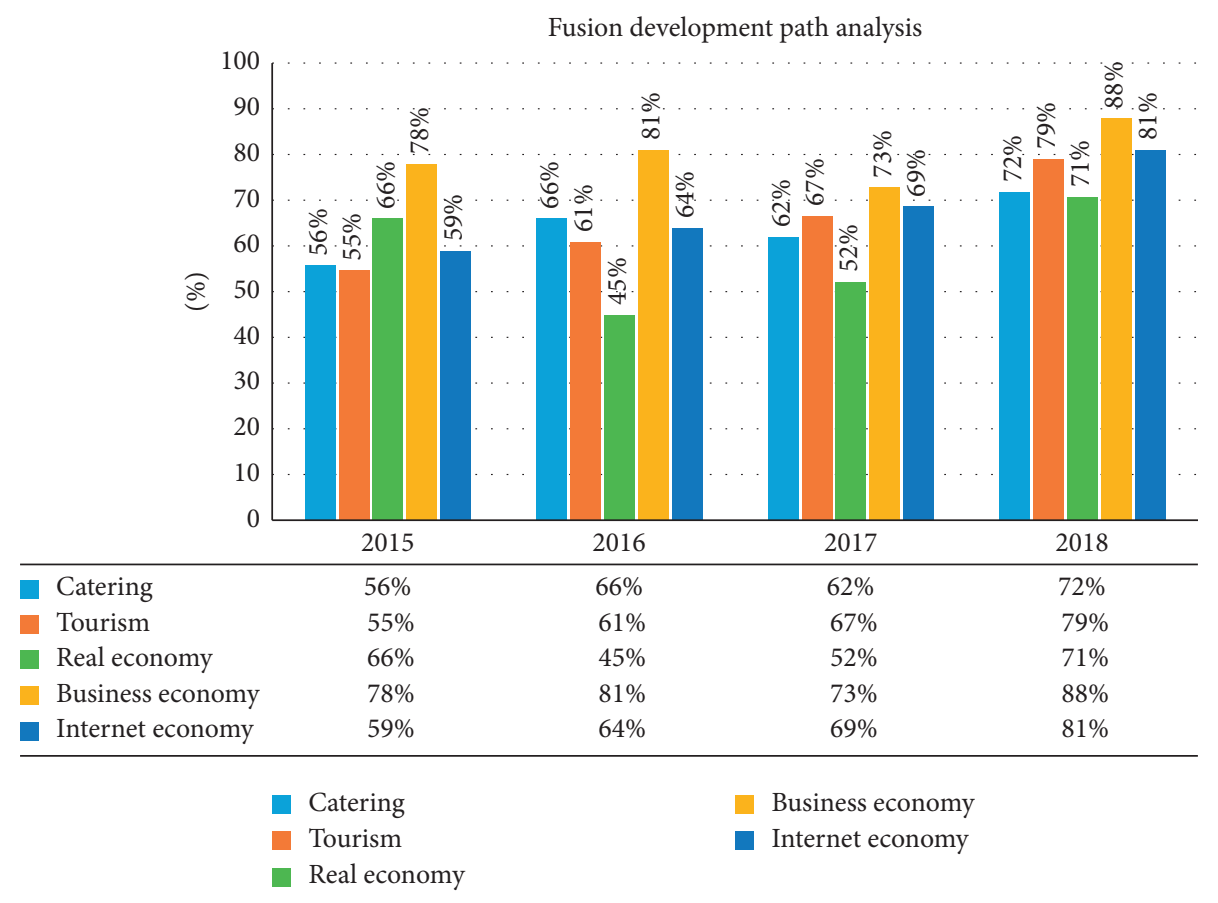

Figure 2: Development path analysis.

just one point in the region. The traffic corridor is the corridor between the points. If the urban agglomeration is regarded as the spatial main body at the macrolevel, the traffic corridor is the network connecting the spatial units of each level and is the basic axis of the spatial diffusion and evolution of the urban agglomeration. Figure 3 is a statistical analysis of the demand analysis of the platform for regional economic development.

As can be seen from the figure, the platform users have the highest demand for public transportation; the percentage is $31.80 \%$; the demand for public energy is second; the demand for public facilities is $21.70 \%$; the smallest is the demand for water, only $18.40 \%$. The layout of transportation facilities affects the evolution of urban form. Throughout the history of urbanization in various countries of the world, the layout of different transportation facilities has a corresponding impact on the spatial and social forms of urban areas. Realize the linear development of traffic corridors, ensure that the vast majority of the employed population in the region can use rail transit, and form an effective radial centripetal commuting mode, which not only ensures the sustainable development of the social economy in the central urban area, but also maintains the original life of urban residents, living habits and effective control of the investment costs of public infrastructure. We can conclude that the layout of transportation facilities affects the evolution of urban morphology and the economic development of human-land industries, and changes in transportation facilities will affect the formation of regional integration. In general, this is because the development of the world's regions and the public transportation system is inseparable. of Regional Integration Industry Economic Development.

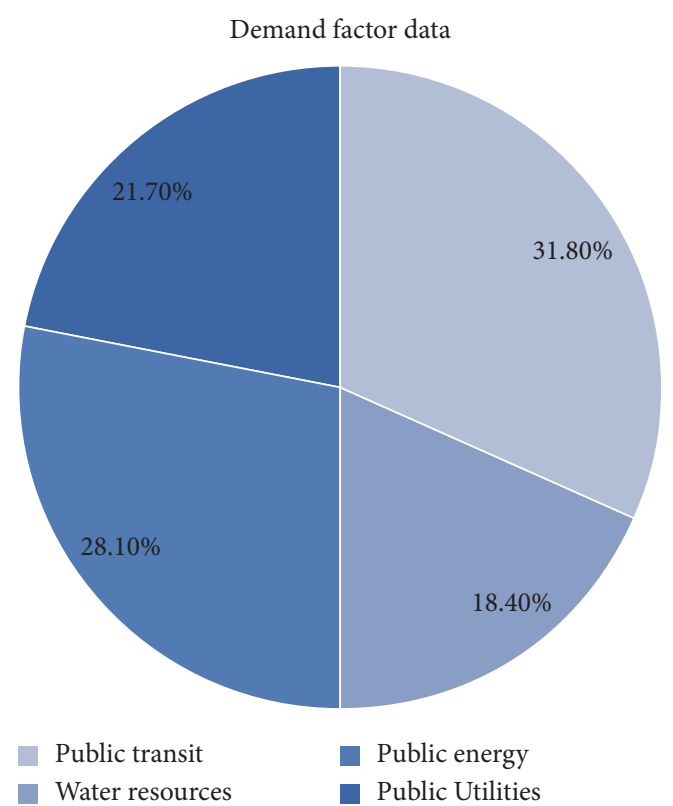

Figure 3: Demand chart.

The relationship between man and land is an objective concept that has existed since the origin of mankind. In the past, human-land relations have always been regarded as the core area of geography research. With the development of human society, the advancement of science and technology, and the improvement of productivity, the connotation of human-land relations has evolved new features. This paper focuses on the perspective of industrial economics of regional integration to analyze and understand the important characteristics of human-land relations in the process of 


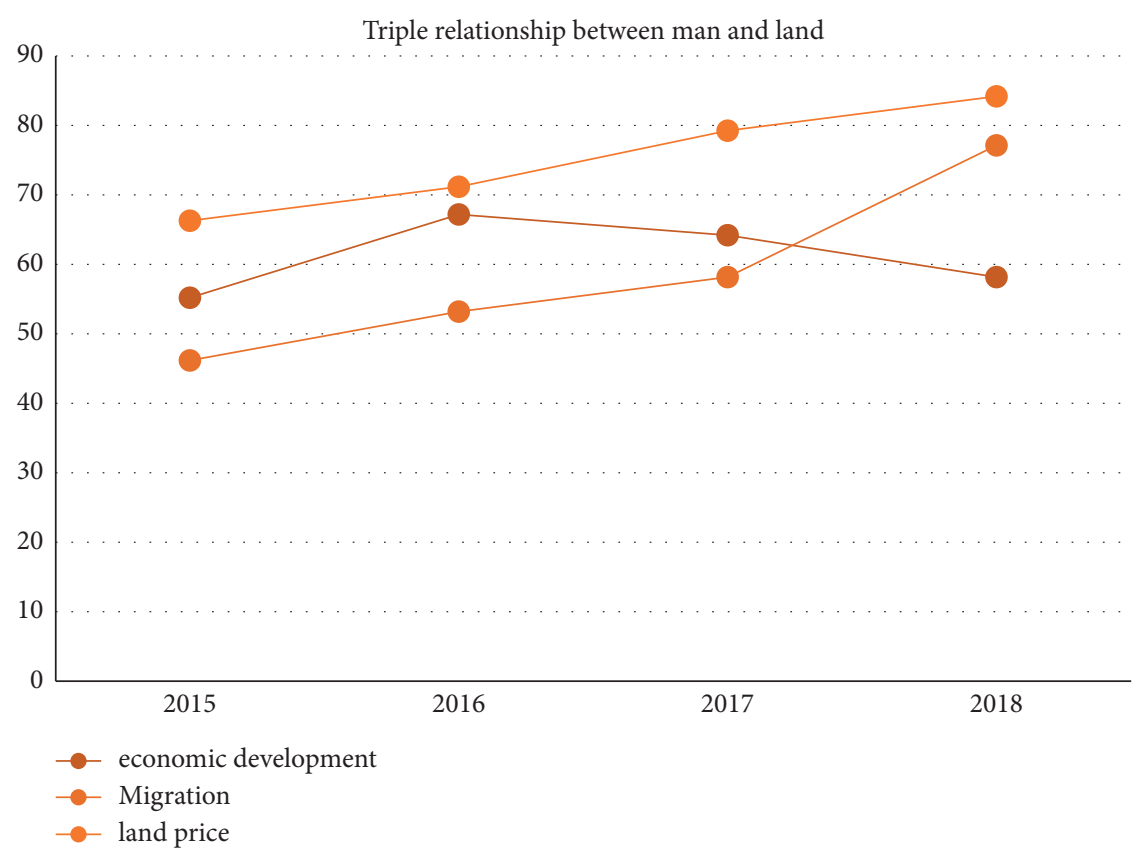

FIGURE 4: Human-land relationship statistics.

regionalization and to predict the regional development trend of industrial economy in the process of industrial integration. Figure 4 shows the analysis and statistics of the platform's tripleness of human-land relationship.

The understanding of the relationship between man and land in the regional economics field mainly refers to the interaction between people who engage in various economic activities or social activities in a specific regional space and the geographical environment in which large geographical differences exist and are constantly changed by human activities. The region is the stage for human social and economic activities, and it is the object for people to develop, utilize, and transform for social progress and self-identity. Since the rapid urbanization in China, the changes in human-land relations have gradually exposed a series of contradictions and problems such as regional environmental coordination, regional social contradictions, regional development imbalances, and uneven distribution of population and resources. To resolve these contradictions and problems, we need to use reasonable regional regulation and balance mechanisms to guide the healthy development of the regional economy.

As shown in the figure, from 2015 to 2018, people's demand for economic development showed a gradual trend, and even a little rebound; in the case of population movement, there was a sudden increase in 2018; and people's price for land was a trend of continuous increase, so land prices are the most concerned aspects of people.

\section{Conclusions}

(1) In the current regional economic development, there is an urgent need to integrate with related technologies in order to adapt to market changes and changes of the times and achieve long-term sustainable development and progress. The research in this paper provides a development idea that integrates with informatization artificial intelligence multimedia technology for future regional economic development and aims to promote the integration of various industries and regional economies and achieve common development in the future development process. The main conclusions of this paper on the economics theory of regional integration industry include the following: First, through the analysis of regional planning practice and theoretical results, it is considered that regional planning has the space to guide economic activities, promote economic development, reshape the law of urban development, and formulate and implement economic characteristics such as economic policy. Secondly, it is proposed that the economics of regional integration industry has multiple characteristics such as geometric form, technicality, management, sociality, and philosophy. Third, it proposes the main research methods of urban planning economics such as investigation and research, analysis and integration, cross-disciplinary research, supply and demand analysis, cost-benefit analysis, marginal analysis, and externalities. Fourth, the main research objectives of urban planning economics include the third "change" that leads the urban planning theory, assisting in the selection, formulation, and management of urban planning schemes, as well as the core values of "people-oriented" urban planning. Fifth, through the study of urban planning and economics related theories, urban planning economics at least includes theoretical factors such as 
cultural economy, land economy, industrial economy, housing economy, public economy, environmental economy, population economy, and regional economy. Sixth, a comprehensive study of the main theoretical research elements of urban planning economics is carried out. It is believed that the above eight major theoretical research elements are closely related to the development of new urbanization. That is to say, the combination of cultural economy and industrial economy can not only achieve the progress of human culture and civilization, but also promote the transformation and upgrading of industrial structure; the integration of public economy and environmental economy can improve the level of urban and rural infrastructure construction, improve the efficiency of economic development, and improve urban and rural areas. The development of land economy and housing economy is conducive to intensive use of land to meet the basic needs of migrants to live in peace; the development of population economy and regional economic competition can further promote regional coordination and enhance the fairness of resource allocation and rationality, providing a multichoice population urbanization implementation path.

(2) In recent years, the performance of regional economic integration demonstrated by the substantive cooperation of governments at all levels in the economic regions represented by the Yangtze River Delta and the Pearl River Delta has also given us enough reason to believe in the construction of an effective region. The government cooperation mechanism to promote and realize regional economic integration and then realize the formation of a unified national market and then coordinate the integration of urban and rural areas is by no means an unreachable utopia.

\section{Data Availability}

This article does not cover data research. No data were used to support this study.

\section{Conflicts of Interest}

The authors declare that they have no conflicts of interest.

\section{Acknowledgments}

This study is a part of a Major Project of Higher Education Teaching Reform in Fujian Province "Professional Certification Based Research on the Construction of National FirstClass Undergraduate Major of International Economy and Trade in Local Applied Universities" (FBJG20200309).

\section{References}

[1] X. Fang, "Study on the development path of regional industrial economic integration based on the construction of smart city," Time Economics and Trade, no. 6, pp. 40-41, 2019.
[2] Z. Cao, "An empirical study on the development of smart city construction and regional industrial economic integration under economic transformation," Contemporary Economics, no. 22 , pp. 88-89, 2018.

[3] H. Niu, "Research on the development of smart city construction and regional industrial economy integration," China Market, no. 28, p. $64+66,2018$.

[4] X. Lan and J. Chen, "The development of smart city construction and regional industrial economy integration," Chinese and Foreign Entrepreneurs, no. 30, pp. 29-30, 2017.

[5] Z. Lin, "Study on the structural transformation of China's industrial economy under the new normal," Contemporary Economics, no. 8, pp. 22-23, 2017.

[6] M. Liu and Y. Cai, "Study on the locational advantage measurement and transformation development path of fujian industrial area," Development Research, no. 10, pp. 73-77, 2016.

[7] Y. Jiang, "Exploring the development model of industrial economy and new media integration," Commercial Economy, no. 9, pp. $64-65+99,2018$.

[8] H. Zhang, "Study on the development path of tangshan ceramic art innovation and industrial economy integration," Industrial Technology and Vocational Education, vol. 15, no. 3, pp. 45-47, 2017.

[9] W. Dong, "Study on the integration of paper industry and internet economy," Economic Research Guide, no. 27, pp. 45-46, 2017.

[10] M. Wang, "Joint, convergence and integration to create a new management pattern-taking the quanzhou evening news agency series industry economic brand promotion activities as an example," News Front, no. 13, pp. 93-94, 2017.

[11] Q. Wang, "Exploration of the development mode of industrial economy and new media integration," China Newspapers, no. 9, pp. 42-43, 2017.

[12] X. Wu, "Discussion on the correlation between informatization and industrial economic growth," Business, no. 35, p. $287,2016$.

[13] W. Zhao, "Constructing a provincial support platform for county-level media centers-changjiang cloud creates a new ecology of regional media convergence," News Front, no. 3, pp. 22-24, 2019.

[14] Z. Zhang, Y. Wang, and Y. Wang, "Strategy of university students' informatization and rural regional economic integration," Jilin Agriculture, vol. 444, no. 3, p. 91, 2019.

[15] W. Cai, On the Integration of National Traditional Sports Culture and Regional Tourism, China National News, Beijing, People's Republic of China, 2019.

[16] M. Zang, "Aiming at the development of leading-edge construction, releasing the new vitality of regional integration," Business Culture, no. 36, pp. 55-57, 2018.

[17] Z. Zhang, "Study on the integration strategy of higher vocational education and regional economic industrial structure connection," Knowledge Economy, no. 23, pp. 101-102, 2018.

[18] E. Mohamed, "The relation of artificial intelligence with internet of things: a survey," Journal of Cybersecurity and Information Management, vol. 1, no. 1, 2020.

[19] N. N. Hurrah, S. A. Parah, N. A. Loan, J. A. Sheikh, M. Elhoseny, and K. Muhammad, "Dual watermarking framework for privacy protection and content authentication of multimedia," Future Generation Computer Systems, vol. 94, pp. 654-673, 2019.

[20] L. Zhu, "Analysis of the integration of information technology and regional economy in chengdu," China Informatization, no. 11, pp. 79-80, 2018. 
[21] Y. Shao, W. Zhang, and Y. Bao, "Study on the development path of the "Belt and road initiative" and regional economic integration," Financial Economy, no. 20, pp. 49-50, 2018.

[22] T. Yu and H. Cao, "Building regional public brands and agricultural pastoral complexes to promote the integration and development of agricultural parks," Grassroots agricultural technology promotion, vol. 6, no. 10, pp. 66-67, 2018.

[23] Z. Lv, Y. Han, A. K. Singh, G. Manogaran, and H. Lv, "Trustworthiness in industrial IoT systems based on artificial intelligence," IEEE Transactions on Industrial Informatics, no. 99 , p. 1, 2020.

[24] S. Gao, "Bottlenecks and innovation paths in the transformation of regional media integration," Contemporary Communication, vol. 202, no. 5, pp. 55-58, 2018.

[25] Y. Zhang, Q. He, Y. Xiang et al., "Low-cost and confidentiality-preserving data acquisition for internet of multimedia things," IEEE Internet of Things Journal, vol. 5, no. 5, pp. 3442-3451, 2017.

[26] Y. Wang, "Study on the thought of regional integration economic development under the background of multimedia," Science and Technology of Energetic Materials, no. 12, pp. 64-65, 2016.

[27] Y. Li and D. Tian, "Research on the influence of multimedia network on economic development," China Market, vol. 992, no. 1, pp. 190-191, 2019.

[28] J. Chen, X. Wang, and Z. Chu, "Capacity sharing, product differentiation and welfare," Economic Research-Ekonomska Istraživanja, vol. 33, no. 1, pp. 107-123, 2020.

[29] Z. Li and W. Xueying, "The realistic bottlenecks and countermeasures of China's real economy transformation and upgrading - based on the analysis of three levels of enterprise, industry and economy," Journal of Xinyang Teachers College(Philosophy and Social Sciences Edition), pp. 1-9.

[30] D. Wu and Y. Ma, "An empirical study on the influence of the scale of graduate education in China on the economic development of the tertiary industry," Degree and Postgraduate Education, no. 2, pp. 18-23, 2019. 